\title{
Changes in the Distribution of Capsular Serotypes of Streptococcus pneumoniae Isolated from Adult Respiratory Specimens in Japan
}

\author{
Hisashi Shoji ${ }^{1}$, Masayuki Maeda ${ }^{2}$, Tetsuro Shirakura ${ }^{3}$, Takahiro Takuma ${ }^{1}$, \\ Hideaki Hanaki ${ }^{4}$ and Yoshihito Niki ${ }^{1}$
}

\begin{abstract}
Objective The objective of this study was to assess whether the distribution of pneumococcal capsular types has been changed, while also providing basic data on changes in the distribution after the introduction of Pneumococcal conjugated vaccine (PCV)13 in adult medical practice.

Methods We analyzed 431 Streptococcus pneumoniae strains (200 in 2006 and 231 in 2012) that had been isolated from respiratory infection specimens from adult patients. Capsular typing was performed by the Quellung reaction and multiplex polymerase chain reaction.

Results A comparison of the 2006 and 2012 strains revealed that the number and proportion of strains by serotype increased from $30(15 \%)$ to $46(20 \%)$ for serotype 3, from $4(2 \%)$ to $14(6 \%)$ for serotype $6 \mathrm{~A}$, and from $4(2 \%)$ to $13(6 \%)$ for serotype $6 \mathrm{C}$, whereas the number and proportion of strains by serotype decreased from $8(4 \%)$ to $0(0 \%)$ for serotype 4 and from $24(12 \%)$ to $17(7 \%)$ for serotype 6B. From 2006 to 2012, the coverage rate significantly decreased from 39 to $28.1 \%$ for PCV7 ( $\mathrm{p}=0.017$ ).

Conclusion Our study showed a decrease in the vaccine coverage of PCV7. However, PCV13 covered serotypes 3 and $6 \mathrm{~A}$, which are prevalent, as well as penicillin-resistant $S$. pneumoniae strains. At present, PCV 13 in adult clinical practice seems to be highly significant. However, there is a possibility that the distribution has changed, and careful screening should be continued in the future.
\end{abstract}

Key words: Streptococcus pneumoniae, pneumococcal polysaccharide vaccine, pneumococcal conjugate vaccine, community-acquired pneumonia, invasive pneumococcal disease

(Intern Med 54: 1337-1341, 2015)

(DOI: 10.2169/internalmedicine.54.3845)

\section{Introduction}

Despite remarkable progress in the clinical practice for pneumonia, the disease remains the third leading cause of death in Japan. Mortality due to pneumonia is particularly increasing rapidly amongst the elderly. The most important factor related to this phenomenon is the aging of society. Other possible factors include inflation in medical costs, an increasing number of antimicrobial-resistant bacteria, and slow development of new antimicrobial agents. There are no practical solutions to address these factors. Pneumococcus is the most important pathogen causing adult respiratory infections (1). S. pneumoniae is a gram-positive bacterial pathogen that causes respiratory and otolaryngological infections such as pneumonia, tympanitis and sinusitis. It also causes serious invasive diseases such as meningitis and septicemia in all age groups. Pneumococcal strains are covered by polysaccharide capsules, and the capsules are closely related to pathogenicity. These capsules serve as a barrier to prevent

${ }^{1}$ Division of Clinical Infectious Diseases, Department of Medicine, School of Medicine, Showa University, Japan, ${ }^{2}$ Division of Infection Control Sciences, Department of Pharmacotherapeutics, School of Pharmacy, Showa University, Japan, ${ }^{3}$ Department of Microbiology, School of Medicine, Showa University, Japan and ${ }^{4}$ Research Center for Infections and Antimicrobials, Kitasato Institute for Life Science, Kitasato University, Japan

Received for publication September 24, 2014; Accepted for publication January 4, 2015

Correspondence to Dr. Hisashi Shoji, itrshoji@med.showa-u.ac.jp 
Table 1. The Detail of Specimens in this Study Each Year.

\begin{tabular}{lrr}
\hline \multirow{2}{*}{ Characteristics } & Year & \\
\cline { 2 - 3 } & 2006 & 2012 \\
\hline Number of specimens & 200 & 241 \\
Participation number of facilities & 35 & 39 \\
Kind of specimens & $190(95.0 \%)$ & $199(86.1 \%)$ \\
Sputum & $6(3.0 \%)$ & $24(10.4 \%)$ \\
Specimens of trans-tracheal aspiration & $2(1.0 \%)$ & $6(2.6 \%)$ \\
Specimens of bronchoscopy & $2(1.0 \%)$ & $2(0.9 \%)$ \\
Others & & \\
Patient backgrounds & $72(62-80)$ & $72(63-79)$ \\
Age, Median (interquartile range) & & \\
Sex & $142(71.0 \%)$ & $153(66.2 \%)^{*}$ \\
Men & $58(29.0 \%)$ & $76(32.9 \%)^{*}$ \\
Female & & \\
Diagnosis & $124(62.0 \%)$ & $162(70.1 \%)$ \\
Community-acquired pneumonia & $26(13.0 \%)$ & $19(8.2 \%)$ \\
Hospital-acquired pneumonia & $27(13.5 \%)$ & $24(10.4 \%)$ \\
Acute Exacerbation of Chronic Bronchitis & $23(11.5 \%)$ & $26(11.3 \%)$ \\
Others & & \\
Susceptibility & $122(61.0 \%)$ & $146(63.2 \%)^{*}$ \\
Penicillin-susceptible $S$. pneumoniae & $70(35.0 \%)$ & $73(31.6 \%)^{*}$ \\
Penicillin-intermediate $S$. pneumoniae & $8(4.0 \%)$ & $11(4.8 \%)^{*}$ \\
Penicillin-resistant $S$. pneumoniae & & \\
* lack of data & &
\end{tabular}

human complement involved in the opsonization from attaching to the bacterial surface. This function gives the bacteria strong power to resist phagocytosis by human polymorphonuclear leukocytes. The capsule thus constitutes the principal cause of the strong pathogenicity of pneumococcal strains. At present, 93 different types of pneumococcal capsule have been identified (2).

Preventive measures against pneumococcal infections are important for carriers who are at a high risk for death due to an immunocompromised condition associated with anticancer agents, corticosteroids, and/or biologicals, human immunodeficiency virus infection or acquired immunodeficiency syndrome. Pneumococcal vaccination is an effective preventive method against pneumococcal infections. Pneumococcal vaccines are classified into two categories, namely, pneumococcal polysaccharide vaccine (PPV) and pneumococcal conjugate vaccine (PCV). Pneumococcal conjugate vaccine, showing higher immunogenicity than PPV, is mainly used in children (3). In the pediatric field, children have been immunized with PCV7, which contains seven types of capsular antigen, since 2010 and PCV13, which contains 13 types, since 2013. The efficacy of PCV7 has been demonstrated in the United States, as represented by a significant reduction in invasive pneumococcal diseases (IPDs) (4).

In Japan, where pneumococcal polysaccharide vaccine 23 (PPV23) containing 23 types of capsular antigen has been conventionally given to adults, PCV13 has become available for adult use since June 2014. In the pediatric field, it has been reported that vaccine coverage rates decreased in association with the changes in the distribution of capsular types after the introduction of the new vaccines (5). We herein report a study of the distribution of capsular serotypes of Streptococcus pneumoniae in the field of adult respiratory infections in Japan, including analysis at the two assessment points of 2006 and 2012. The study is aimed to assess whether the distribution has been changed and also to provide basic data to assess changes in the distribution after the introduction of PCV13 in adult medical practice.

\section{Materials and Methods}

\section{Streptococcus pneumoniae strains}

In this study, we obtained 200 and 231 Streptococcus pneumoniae strains from a Japanese surveillance in 2006 and 2012, respectively. The surveillance was nationwide and conducted by the Japanese Society of Chemotherapy, the Japanese Association for Infectious Diseases and the Japanese Society of Clinical Microbiology (6). The strains of well-diagnosed adult respiratory tract infection were isolated from the sputum and specimens of the trans-tracheal aspiration or bronchoscopy $(1,6)$. Their causality was confirmed by quantitative culture, Gram staining, and observation of phagocytes $(1,6)$. Table 1 shows the participation number of institutes, patient backgrounds and kind of specimens. All strains were grown on Sheep Blood Agar ${ }^{\circledR}$ (Nippon Beckton-Dickinson, Tokyo, Japan) and maintained at $35^{\circ} \mathrm{C}$ with $5 \% \mathrm{CO}_{2}$. The strains were stored in a Microbank ${ }^{\mathrm{TM}}$ system (IWAKI, Tokyo, Japan) at $-80^{\circ} \mathrm{C}$.

\section{Susceptibility testing}

The bacterial susceptibility to penicillin G (PCG) was studied using the broth microdilution method. The reference broth microdilution method was performed according to the Clinical Laboratory Standard Institution guidelines (7). The test medium was prepared using cation-adjusted MuellerHinton broth (Eikenkagaku, Tokyo, Japan) with lysed horse blood (Nippon Biotest Laboratory, Tokyo, Japan). Microtiter plates were then inoculated to produce a final inoculum density of approximately $5 \times 10^{5}$ colony forming unit $(\mathrm{CFU}) / \mathrm{mL}$, which was regularly controlled by counting the colonies. The inoculated plates were incubated at $36^{\circ} \mathrm{C}$ for $20-24 \mathrm{~h}$ before interpreting the results. Minimum inhibitory consentration (MIC) was defined as the lowest concentration of antibiotics that inhibited bacterial growth. Strains with PCG MIC $\leq 0.06 \mu \mathrm{g} / \mathrm{mL}$ were classified as penicillin-susceptible $S$. pneumoniae (PSSP), those with PCG MIC of 0.12 to $1 \mu \mathrm{g}$ / $\mathrm{mL}$ as penicillin-intermediate $S$. pneumoniae (PISP), and those with PCG MIC $\geq 2 \mu \mathrm{g} / \mathrm{mL}$ as penicillin-resistant $S$. pneumoniae (PRSP).

\section{Capsular typing}

Capsular typing was performed by the Quellung reaction and multiplex polymerase chain reaction (PCR). The Quellung reaction was performed using a set of antisera obtained from Statens Serum Institute (Copenhagen, Denmark). The typing procedure was performed as described in the previous report (8). The multiplex PCR was performed based on the procedure of the Streptococcus Laboratory in the Center for Disease Control and Prevention (9). The PCRs were per- 


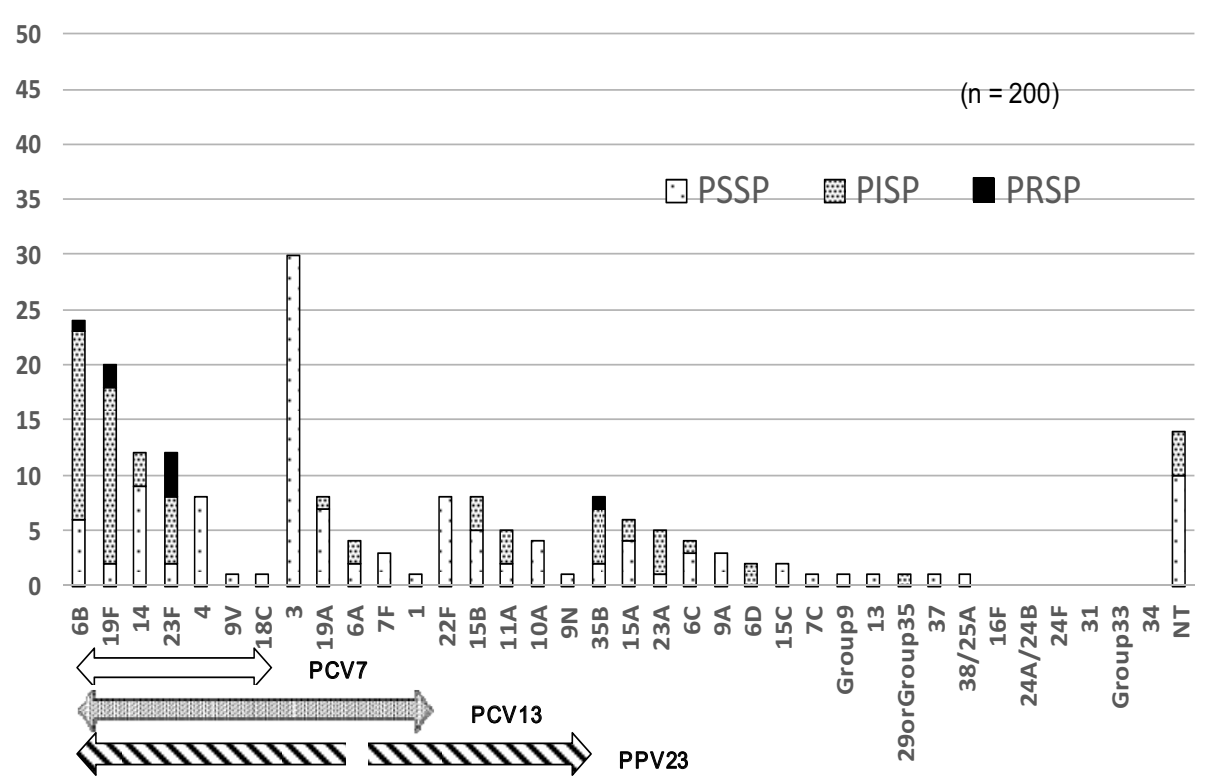

Figure 1. Distribution of serogroups/types amongst 200 Streptococcus pneumoniae isolated from adult patients with respiratory infections in 2006. PSSP: penicillin-susceptible $S$. pneumoniae, PISP: penicillin-intermediate $S$. pneumoniae, PRSP: penicillin-resistant $S$. pneumoniae

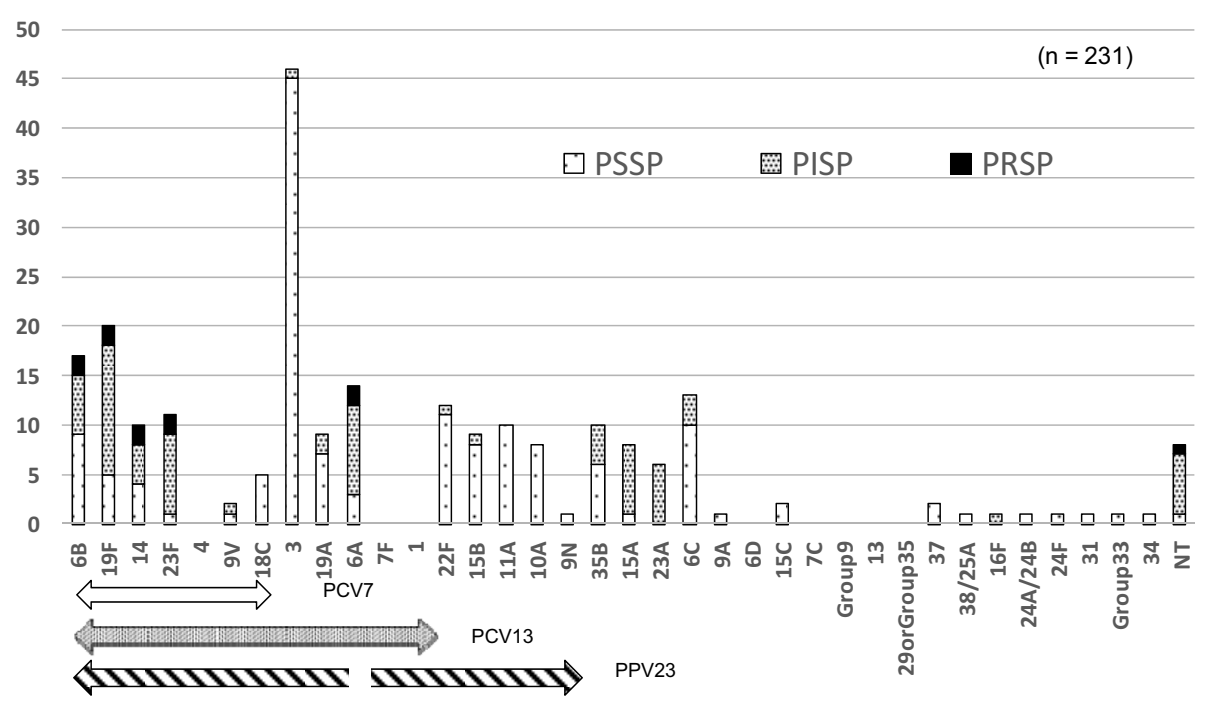

Figure 2. Distribution of serogroups/types amongst 231 Streptococcus pneumoniae isolated from adult patients with respiratory infections in 2012. PSSP: penicillin-susceptible $S$. pneumoniae, PISP: penicillin-intermediate $S$. pneumoniae, PRSP: penicillin-resistant $S$. pneumoniae

formed in $25 \mu \mathrm{L}$ volumes, and each reaction mixture contained the following: $2 \times$ PCR buffer (QIAGEN Multiplex PCR Kit, Hilden, Germany) $12.5 \mu \mathrm{L}$, clinical sample of DNA $5 \mu \mathrm{L}$, and primers with concentrations as specified in the protocol published by the Centers for Disease Control and Prevention.

\section{Results}

Figs. 1 and 2 show the distribution of the serotypes of the analyzed isolates. Among all 431 strains pooled from the 2006 and 2012 surveys, serotype 3 was the most common and was identified from the 76 strains, followed by sero- types $6 \mathrm{~B}, 19 \mathrm{~F}, 23 \mathrm{~F}$, and 14 . A comparison of the data of 2006 with those of 2012 revealed that the number and proportion of the strains by serotype increased from $30(15 \%)$ to $46(20 \%)$ for serotype 3 , from $4(2 \%)$ to $14(6 \%)$ for serotype $6 \mathrm{~A}$, and from $4(2 \%)$ to $13(6 \%)$ for serotype $6 \mathrm{C}$, whereas the number and proportion of strains by serotype decreased from $8(4 \%)$ to $0(0 \%)$ for serotype 4 and from $24(12 \%)$ to $17(7 \%)$ for serotype 6B. Table 2 shows the vaccine coverage rates by vaccine type in 2006 versus 2012 . From 2006 to 2012, the coverage rate significantly decreased from 39 to $28.1 \%$ for PCV7 ( $\mathrm{p}=0.017)$, and tended to decrease from 62 to $58.4 \%$ for PCV13, and from 73 to $69.7 \%$ for PPV23. 
Table 2. Vaccine Coverage Rates by Vaccine Type in 2006 Versus 2012.

\begin{tabular}{lrrr}
\hline \multirow{2}{*}{ Vaccine type } & \multicolumn{2}{l}{ Year } & \multirow{2}{*}{ p value $^{*}$} \\
\cline { 2 - 3 } & 2006 & 2012 & \\
\hline PCV7 & $39.0 \%$ & $28.1 \%$ & 0.017 \\
PCV13 & $62.0 \%$ & $58.4 \%$ & 0.452 \\
PPV23 & $73.0 \%$ & $69.7 \%$ & 0.450 \\
\hline "Chi-square test & & &
\end{tabular}

Regarding the number and proportion of strains by penicillin susceptibility, the 200 strains from the 2006 survey consisted of 122 PSSP strains (61\%), 70 PISP strains (35\%), and 8 PRSP strains $(4 \%)$, while the 231 strains from the 2012 survey consisted of 147 PSSP strains (64\%), 73 PISP strains $(32 \%)$, and 11 PRSP strains (4\%). No significant changes in penicillin susceptibility were found over the time course. The 16 incremental strains of serotype 3 that increased from 2006 to 2012 were 15 PSSP strains and 1 PISP strain. The 10 incremental strains of serotype $6 \mathrm{~A}$ were 1 PSSP strain, 7 PISP strains, and 2 PRSP strains. As a result, the vaccine coverage rate of PRSP was $88 \%$ (7/8 strains) for all vaccine types of PCV7, PCV13, and PPV23 in 2006, compared with 73\% (8/11 strains) for PCV7 and PPV23, and 91\% (10/11 strains) for PCV13 in 2012.

\section{Discussion}

We studied changes in the distribution of the capsular serotypes of Streptococcus pneumoniae in the field of adult respiratory infections in Japan. The distribution partially differed from the distribution in the field of pediatric IPDs. In the field of adult respiratory infections, serotype 3 was the most common, followed by serotypes $6 \mathrm{~B}, 14,19$, and $23 \mathrm{~F}$. In the field of pediatric IPDs, however, serotype 3 was not common, and other serotypes, namely, 6B, 14, 19, and 23F were frequently isolated, as in the field of adult respiratory infections (10). These findings suggest a relative close relationship in the origin of pneumococcal strains isolated from adults and from children. In the present study, the numbers of serotypes $6 \mathrm{~B}$ and 4 strains markedly decreased from 2006 to 2012, thus resulting in a decrease in the vaccine coverage of PCV7. The serotype 3 is the most frequently isolated in Japan. Furthemore the serotype 3 is often isolated from empyema, necrotizing pneumonia, septic shock and meningitis, and leads to severe clinical outcomes (11). In the United States, changes in the capsular serotypes distribution (included 3) after the introduction of new vaccines in the pediatric field resulted in a reduced vaccine coverage $(5,12)$. In Japan, PCV7 was introduced in the pediatric field in 2010. Since our study was not designed to analyze the capsular serotypes in the pediatric field, it is unclear whether the distribution of the capsular serotypes in children has changed or not. However, a decrease in the vaccine coverage of PCV7 found in adult respiratory infections may be somehow related to the introduction of PCV7 in the pediatric field.

Pneumococcal polysaccharide vaccine 23 , which was con- ventionally given to adults, is prepared using capsular polysaccharides as antigens. A capsular polysaccharide acts as a $\mathrm{T}$ cell-independent antigen, which induces the $\mathrm{B}$ cells to produce antibodies without $\mathrm{T}$ cell assistance. Capsular serotype-specific immunoglobulin $\mathrm{G}$ antibodies produced by $\mathrm{B}$ cells bind to the bacteria to exert opsonic activity and thereby help the body eliminate the bacteria. However, immunity acquired against $\mathrm{T}$ cell-independent antigens diminishes within several years owing to the absence of established immunological memory and cannot be boosted by additional vaccination. In infants aged two years and younger, the clinical efficacy of PPV23 cannot be expected due to low immunogenicity (13). Children have been immunized with PCV7 containing 7 types of capsular antigens since 2010, and PCV13 containing 13 types since 2013. These new vaccines are prepared with capsular saccharides conjugated to cross-reacting material 197 (CRM 197) of nonpathogenic diphtheria as the carrier protein to convert them to $T$ cell antigens. They reportedly induce an adequate immune response by their strong immunogenicity in infants aged two years and younger (3).

In Japan, PCV13 vaccination in adults became available in June 2014. Our study demonstrated that PCV13 covers serotypes 3 and $6 \mathrm{~A}$, which are increasing in prevalence, as well as PRSP strains. In addition, there is a report that the serotype 3 has high pathogenicity (11). At present, the introduction of PCV13 in adult clinical practice seems to be highly significant. However, changes in the distribution of the capsular serotypes have been reported after the introduction of PCV13, as well as PCV7 (14). This indicates that careful screening should be continued in the future.

In the present study, there was not a significant change in the coverage rate for PPV23, which was 73\% in 2006 and $69.7 \%$ in 2012. It is not known which vaccine is the most appropriate for adults, either a vaccine that covers a broad range of capsular serotypes, or a vaccine with increased immunogenicity. Further studies should therefore be continued in the future.

\section{Author's disclosure of potential Conflicts of Interest (COI).}

Hisashi Shoji: Research funding, Takeda Pharmaceutical, Pfizer Japan, Daiichi Sankyo, Bayer Yakuhin, Taisho Toyama Pharmaceutical, Dainippon Sumitomo Pharma, Mitsubishi Tanabe Pharma, Kyorin Pharmaceutical, Astellas Pharma, Chugai Pharmaceutical and GlaxoSmithKline. Takahiro Takuma: Research funding, Takeda Pharmaceutical, Pfizer Japan, Daiichi Sankyo, Bayer Yakuhin, Taisho Toyama Pharmaceutical, Dainippon Sumitomo Pharma, Mitsubishi Tanabe Pharma, Kyorin Pharmaceutical, Astellas Pharma, Chugai Pharmaceutical and GlaxoSmithKline. Yoshihito Niki: Research funding, Takeda Pharmaceutical, Pfizer Japan, Daiichi Sankyo, Bayer Yakuhin, Taisho Toyama Pharmaceutical, Dainippon Sumitomo Pharma, Mitsubishi Tanabe Pharma, Kyorin Pharmaceutical, Astellas Pharma, Chugai Pharmaceutical and GlaxoSmithKline. 


\section{References}

1. The committee for the Japanese Respiratory Society guidelines in management of respiratory infections. The Japanese Respiratory Society guideline for the management of community-acquired pneumonia in adults. Respirology 11(Suppl 3): S79-S133, 2005.

2. Austrian R, Douglas RM, Schiffman G, et al. Prevention of pneumococcal pneumonia by vaccination. Trans Assoc Am Physicians 89: 184-194, 1976.

3. Black S, Shinefield H, Baxter R, et al. Impact of the use of heptavalent pneumococcal conjugate vaccine on disease epidemiology in children and adults. Vaccine 24 (Suppl 2): S2-79-80, 2006.

4. Centers for Disease Control and Prevention (CDC). Invasive pneumococcal disease in children 5 years after conjugate vaccine introduction--eight states, 1998-2005. MMWR Morb Mortal Wkly Rep 57: 144-148, 2008.

5. Pilishvili T, Lexau C, Farley MM, et al. Sustained reductions in invasive pneumococcal disease in the era of conjugate vaccine. $\mathrm{J}$ Infect Dis 201: 32-41, 2010.

6. Niki Y, Hanaki H, Yagisawa M, et al. The first nationwide surveillance of bacterial respiratory pathogens conducted by the Japanese Society of Chemotherapy. Part 1: a general view of antibacterial susceptibility. J Infect Chemother 14: 279-290, 2008.

7. The Clinical and Laboratory Standards Institute (CLSI). Methods for dilution antimicrobial susceptibility tests for bacteria that grow aerobically; approved standard. In: Clinical and Laboratory Standards Institute. 7th ed. Wayne, PA, 2006: M7-A7.
8. Sørensen UB. Typing of pneumococci by using 12 pooled antisera. J Clin Microbiol 31: 2097-2100, 1993.

9. Centers for Disease Control and Prevention. PCR Deduction of Pneumococcal Serotypes [Internet]. [cited 2013 Dec 16]. Available from: http://www.cdc.gov/streplab/pcr.html

10. Chiba N, Morozumi M, Shouji M, Wajima T, Iwata S, Ubukata K. Changes in capsule and drug resistance of Pneumococci after introduction of PCV7, Japan, 2010-2013. Emerg Infect Dis 20: 1132-1139, 2014.

11. Grabenstein JD, Musey LK. Differences in serious clinical outcomes of infection caused by specific pneumococcal serotypes among adults. Vaccine 32: 2399-2405, 2014.

12. Mendes RE, Costello AJ, Jacobs MR, Biek D, Critchley IA, Jones RN. Serotype distribution and antimicrobial susceptibility of USA Streptococcus pneumoniae isolates collected prior to and post introduction of 13-valent pneumococcal conjugate vaccine. Diagn Microbiol Infect Dis 80: 19-25, 2014.

13. Timens W, Boes A, Rozeboom-Uiterwijk T, Poppema S. Immaturity of the human splenic marginal zone in infancy. Possible contribution to the deficient infant immune response. J Immunol 143: 3200-3206, 1989.

14. Mendes RE, Costello AJ, Jacobs MR, Biek D, Critchley IA, Jones RN. Serotype distribution and antimicrobial susceptibility of USA Streptococcus pneumoniae isolates collected prior to and post introduction of 13-valent pneumococcal conjugate vaccine. Diagn Microbiol Infect Dis 80: 19-25, 2014.

(C) 2015 The Japanese Society of Internal Medicine http://www.naika.or.jp/imonline/index.html 